\title{
Ward Round - a boy with multiple joint swellings
}

\section{David Tickell}

Dept of Paediatrics, Queen Elizabeth Central Hospital, Blantyre

IK is a 10-year-old HIV non-reactive boy who was referred to Queen Elizabeth Central Hospital (QECH) General Paediatric Clinic in early October 2007 with complaints of several months of multiple joint swellings and loss of range of movement. We could not identify any obvious trigger for the joint problems. There were no associated systemic features such as fever, lethargy, malaise or rash.

He was a thin, rather despondent looking child with tender, boggy, non-erythematous but mildly warm swelling of both ankles (figure 1), knees (figure 2), elbows and wrists as well as several proximal interphalangeal (PIP) joints bilaterally (figure 3). All of these joints were tender and had a reduced range of movement, and he found it difficult and painful to walk. There was no rash or fever, no obvious eye changes, no lymphadenopathy and a normal abdominal examination.

He had been managed at a nearby hospital for the previous month with prednisolone $5 \mathrm{mg}$ twice daily and regular ibuprofen (200mg tds) for a presumed inflammatory process, as well as flucloxacillin for a potential infective component. There had not been much change in his symptoms over this time.

IK's family, including his parents, two brothers and a sister, all live in Nsanje where his father works as a computer software installer. The other family members were all well and IK himself had not suffered from any other significant medical conditions in the past.

\section{What is the likely diagnosis?}

\section{What management steps would you institute?}

What would be your further management if his initial response was poor?

\section{Continued on page 101}

Figure 1 swelling of both ankles
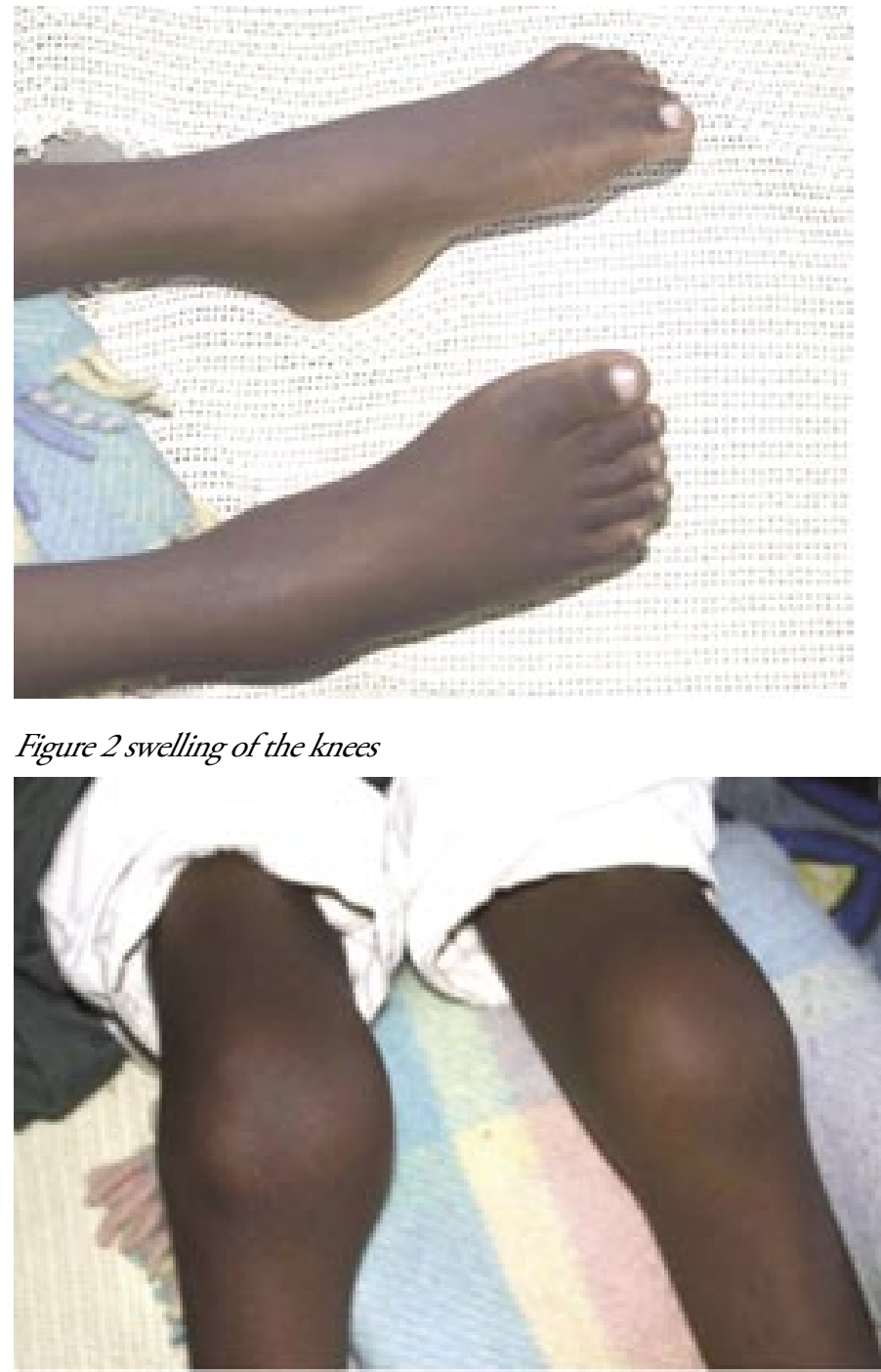

Figure 3

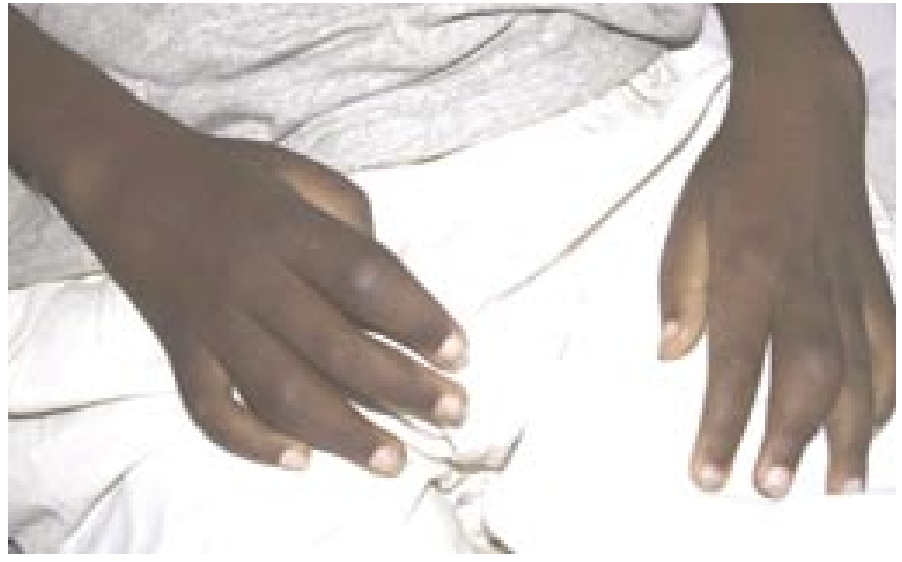




\section{Ward Round - A boy with multiple joint swellings \\ Continued from page 99}

$\mathrm{X}$-rays showed evidence of osteomyelitis involving almost Case discussion of patient IK

We made a presumptive diagnosis of polyarticular juvenile idiopathic arthritis (JIA).

The child was commenced on a higher-dose course of prednisolone $20 \mathrm{mg}$ daily for 2 weeks, then reduced to $10 \mathrm{mg}$ $\& 5 \mathrm{mg}$ for a further 2 weeks each. Ibuprofen was continued, and we added a proton pump inhibitor to reduce excessive gastric acid secretion that might result from the other drugs. He was also sent for ophthalmological assessment for potential uveitis (although this is more common in oligoarticular JIA) and commenced on some supplementary feeds.

Upon review one month later IK was much improved, with a pain-free gait and significant reduction in the inflammation of his involved joints. He was much happier in himself and was putting on weight (although this may have been a side effect of the steroid therapy as much as the nutritional supplementation). He was maintained on low dose prednisolone (5mg per day) orally as well as his non-steroidal anti-inflammatory drug (NSAID).

Over the next several months IK had a stormy course of recurrent symptom flare-ups requiring higher dosing of prednisolone for their control. He was never able to do without treatment altogether. We considered the possibility of giving steroids directly by intra-articular injection, but this was not a realistic option since so many joints were affected.

We discussed the option of adding a disease modifying antirheumatoid drug (DMARD), methotrexate, to his treatment, and were planning to commence this when IK again presented ankles and several tarsal bones, his right elbow and several PIP joints. He was now almost unable to bear weight. We administered high dose IV pulse steroids, using $1 \mathrm{~g} / \mathrm{m} 2$ dexamethasone on days $1,3 \& 5$. He responded well to this and we then recommenced daily high dose oral steroids, to which we added methotrexate $10 \mathrm{mg} / \mathrm{m} 2$ once weekly. A full blood count was normal prior to commencing the DMARD. We added folic acid to the regimen because methotrexate is a dihydrofolate reductase inhibitor.

After a one month admission with intensive physiotherapy he had improved but was still having great difficulty walking. Before discharge he was given a single slow IV dose of $15 \mathrm{mg} / \mathrm{m} 2$ methotrexate with normal saline hyperhydration and cotrimoxazole prophylaxis, then sent home with an increased dose of $20 \mathrm{mg} / \mathrm{m} 2 /$ week methotrexate, tapering prednisolone dosing, a proton pump inhibitor, a NSAID and folic acid. He had become somewhat Cushingoid in appearance by this time.

IK's father has been told that the methotrexate is 'disease modifying', and therefore that it will take several months to convey its full benefit. He is to be seen weekly in Nsanje, and will travel to the central hospital in Blantyre $\sim 2$-monthly where his symptoms and full blood count will be reviewed.

If funds were available, this boy would be an excellent candidate for early introduction of a biologic diseasemodifying agent (e.g. etanercept, a drug that contains a TNF-binding receptor which modifies TNF's inflammatory effects). Even if it could be afforded, such therapy would only be considered if his response to methotrexate were to prove unsatisfactory in the coming months. 